\title{
Survival outcome among patients with Ewing's sarcoma of bones and joints: a population-based cohort study
}

\author{
Zi-Hao Wan', Zhi-Hao Huang", Liao-Bin Chen"' \\ Zhongnan Hospital, Wuhan University, Wuhan, Hubei Province, China
}

'MD, MSc. Surgeon, Department of Orthopedic Surgery, Zhongnan Hospital, Wuhan University, Wuhan, Hubei Province, China.

"MD, MSc. Surgeon, Department of Colorectal and Anal Surgery, Zhongnan Hospital, Wuhan University, Wuhan, Hubei Province, China.

"'MD. Surgeon, Department of Orthopedic Surgery, Zhongnan Hospital, Wuhan University, Wuhan, Hubei Province, China.

(Dorcid.org/0000-0002-4854-4213

\section{KEY WORDS:}

Bone and bones.

Sarcoma, Ewing

Survival analysis

Retrospective studies.

\begin{abstract}
BACKGROUND: The aim here was to elucidate the current survival condition of patients diagnosed with Ewing's sarcoma of the bones and joints and determine independent risk factors associated with the prognosis. DESIGN AND SETTING: Retrospective cohort study based on the Surveillance, Epidemiology and End Results (SEER) database in the United States.

METHODS: We identified 397 patients who were diagnosed with Ewing's sarcoma of the bones and joints between January 2004 and December 2013. The multivariate Cox proportional hazards model was used to determine factors associated with the risk of death by adjusting for various factors.

RESULTS: The one, two and five-year disease-specific survival rates were $89.08 \%, 78.08 \%$ and $62.47 \%$, respectively. The factors related to death were age $(\geq 18$ years versus $<18$ years; hazard ratio, $H R=1.77$; 95\% confidence interval, $\mathrm{Cl}: 1.38-2.31$ ); tumor site (extremity versus spine and pelvis; $\mathrm{HR}=2.03 ; 95 \% \mathrm{Cl}$ : 1.31-2.62); tumor size ( $>10 \mathrm{~cm}$ versus $\leq 10 \mathrm{~cm} ; \mathrm{HR}=1.78 ; 95 \% \mathrm{Cl}: 1.34-2.56$ ); and type of treatment (surgery alone versus radiotherapy with surgery; $\mathrm{HR}=0.51 ; 95 \% \mathrm{Cl}$ : $0.38-0.89$; or radiotherapy alone versus radiotherapy with surgery; $\mathrm{HR}=1.61 ; 95 \% \mathrm{Cl}: 1.10-2.39$; or no treatment versus radiotherapy with surgery; $\mathrm{HR}=1.86 ; 95 \% \mathrm{Cl}: 1.23,2.58)$

CONCLUSIONS: Patients with Ewing's sarcoma showed poor survival in situations of age $\geq 18$ years, tumor size $>10 \mathrm{~cm}$, receiving radiotherapy alone and receiving no treatment. Patients undergoing surgery alone had better survival.
\end{abstract}

\section{INTRODUCTION}

Ewing's sarcoma is a rare cancer that accounts for less than $10 \%$ of all malignancies existing in the human body. It stems from primitive neuroepithelial cells, which are able to differentiate into various mesenchymal cells, and has a propensity to metastasize to distant sites at an early stage. This cancer typically occurs in adolescents and young adults, accompanied by a very poor prognosis. It is considered to be a high-grade malignancy, ranking second in the list of malignant bone tumors. ${ }^{1-7}$ It is commonly considered to be an extremely aggressive osteolytic cancer that usually occurs in the bones of the limbs and pelvis and it can metastasize to distant locations such as bone marrow, the lungs and other soft tissues at an early stage. ${ }^{1,3,7}$

In the United States, the overall incidence rate of Ewing's sarcoma is approximately 0.1 case per 100,000 individuals per year, and this rate had not undergone any obvious change over past 30 years. An estimated $90 \%$ of these patients are under 20 years old, and the death rate is approximately 0.05 cases per 100,000 individuals per year. Additionally, most cases of Ewing's sarcoma of the bones and joints are found in the limbs, pelvis or spine.,8

Nonetheless, there is a lack of survival studies on Ewing's sarcoma arising in the bones and joints and associated prognostic factors, based on up-to-date data on nationwide populations.

\section{OBJECTIVE}

The purpose of this study was to demonstrate the survival conditions of patients with Ewing's sarcoma of the bones and joints and determine independent risk factors associated with their prognosis.

\section{METHODS}

The Surveillance, Epidemiology and End Results (SEER) database named "Incidence-SEER 18 Regs Research Data + Hurricane Katrina Impacted Louisiana Cases, Nov 2015 Sub (1973-2013 varying)” 
was selected to perform a population-based search for patients suffering from Ewing's sarcoma of the bones and joints between January 2004 and December 2013.

The SEER Program ${ }^{9}$ is supported by the National Cancer Institute of the United States and has provided statistical information on tumor cases since 1973. It collects data on cases diagnosed with cancer throughout the United States, with an estimated $28 \%$ of the United States population covered. The SEER registry is a validated database that is frequently applied for cancer survival studies. The National Cancer Institute does not require institutional review board approval for SEER studies because it is an unidentified public-use database.

Histological International Classification of Diseases (ICD) codes (ICD-0-3) were used to identify Ewing's sarcoma (9260/3), (9364/3). Site-specific codes (C40.0-C40.3, C40.8, C40.9, C41.2, C41.4 and C41.8) were used to screen for tumors originating in the extremities, pelvis and spine, while the codes for bones of the skull and face, mandible, rib, sternum, clavicle and associated joints were not included.

The following primary data were drawn from the database for analysis: age at diagnosis, sex, race, tumor site, tumor size, tumor grade, type of treatment, cause of death and survival in months. Patients diagnosed through either autopsy or the death certificate were excluded. Those who presented secondary malignancies at the time of diagnosis or whose diagnoses were not confirmed by means of histopathological evaluation were also excluded. Cases without complete information were excluded. The inclusion and exclusion procedure is showed in the flow chart of Figure $\mathbf{1 .}$

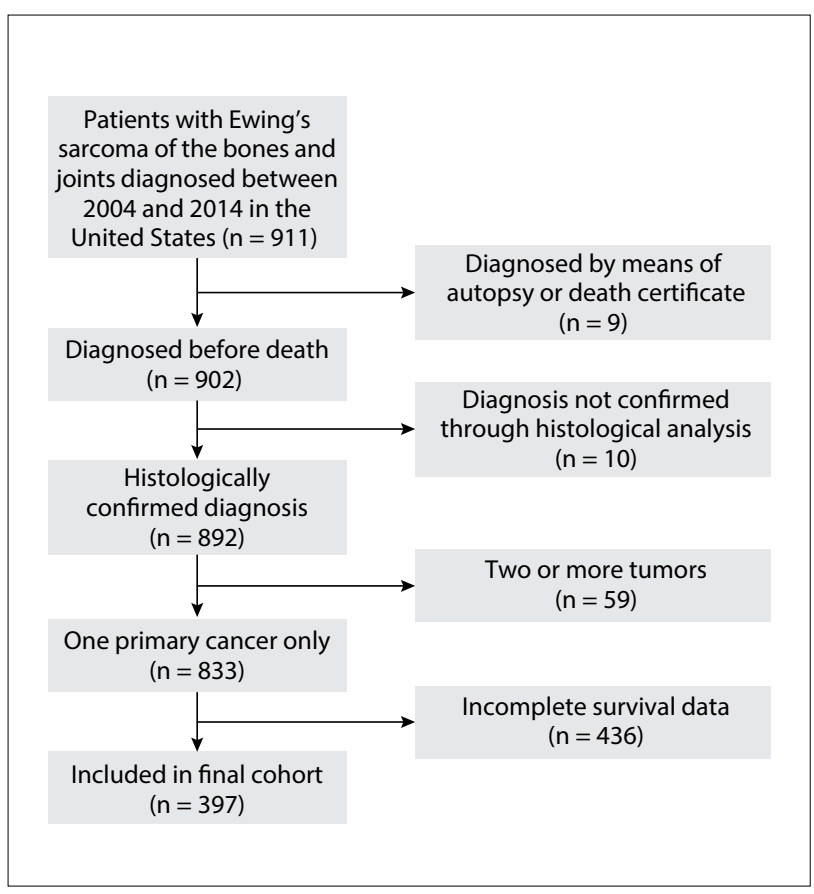

Figure 1. Flow chart for selection of the study cohort.
Well-differentiated and moderately differentiated histological features were classified as low grade while poorly-differentiated and undifferentiated histological types were classified as high grade. The pathological tumor-node-metastasis (pTNM) classification was used in cases of patients who underwent tumor resection, because their tumor gradings could be confirmed through analysis under a microscope. Because the sixth edition of the staging system of the American Joint Commission on Cancer was used in the SEER database starting in 2004 and the most recent update for this database was in 2013 (which was when the present study began), we chose to use this period (2004 to 2013) for the present study.

The main result from this study was the disease-specific survival (DSS). Descriptive statistics were calculated for all factors. The DSS was calculated by means of the Kaplan-Meier method, and the log-rank test was applied to appraise the deviations. We calculated hazard ratios (HRs) and the $95 \%$ confidence interval (CI) in the Cox proportional hazards model after adjusting for various variables. The SEER ${ }^{\star}$ Stat application version 8.3.2 (IMS Inc., USA) was used to extract primary data. All statistical analyses was finished in the SPSS software, version 23 (IBM Corp., USA). Differences between groups were taken to be statistically significant when the $\mathrm{P}$ value was less than 0.05 .

\section{RESULTS}

During the 10-year period, 397 patients diagnosed with Ewing's sarcoma of the bones and joints were included (249 males and 148 females). Table 1 shows the distribution of the patients' characteristics in the study. The mean age (with SD) at diagnosis was 18.5 (12.4) years. $65.1 \%$ of the patients were aged $<18$ years. Tumors arising from the extremities accounted for $55.1 \%$. 378 tumors $(95.2 \%)$ were histologically confirmed to be poorly-differentiated or undifferentiated tumors. The mean tumor size at the time of diagnosis was $10.3 \mathrm{~cm}$ (5.3). 86 patients (21.6\%) received surgery with radiotherapy, 139 (35.1\%) underwent surgical resection alone, 102 (25.7\%) underwent radiotherapy alone and $70(17.6 \%)$ received no treatment.

The overall one, two and five-year survival rates after diagnosis were $89.08 \%, 78.08 \%$ and $62.47 \%$, respectively (Figure 2 ). The five-year relative survival rates were $78.4 \%, 66.9 \%, 47.8 \%$ and $44.8 \%$ for patients receiving surgery, surgery with radiotherapy, radiotherapy alone and no therapy, respectively (Figure 3). Overall, patients with tumor size $\leq 10 \mathrm{~cm}$ had a higher five-year survival rate than did those with tumor size $>10 \mathrm{~cm}(70.8 \%$ versus $52.4 \%$; $\mathrm{P}<0.001$ ) (Figure 4). The five-year survival rate were $68.7 \%$ and $50.2 \%$ for those $<18$ and $\geq 18$ years (Figure 5 ).

Table 2 shows the results from univariate and multivariate Cox proportional hazards analyses. Age $\geq 18$ years $(\mathrm{HR}=1.77$; $95 \% \mathrm{CI}=1.33-2.01)$, tumor originating in the spine and pelvis $(\mathrm{HR}=2.03 ; 95 \% \mathrm{CI}=1.31-2.62)$, tumor size $>10 \mathrm{~cm}(\mathrm{HR}=1.78$; 
$95 \% \mathrm{CI}=1.24-2.35)$, radiotherapy alone $(\mathrm{HR}=1.61 ; 95 \%$ $\mathrm{CI}=1.10-2.39)$ and no treatment $(\mathrm{HR}=1.86 ; 95 \% \mathrm{CI}=1.23-2.58)$ were associated with increased risk of mortality, while receiving surgery alone $(\mathrm{HR}=0.51 ; 95 \% \mathrm{CI}=0.38-0.89)$ was an independent predictor for longer survival.

\section{DISCUSSION}

In our study, we found that the one, two and five-year DSS rates were $89.08 \%, 78.08 \%$ and $62.47 \%$, respectively. These proportions were reported to be higher in a previous investigation. ${ }^{10}$ This difference may reflect that the prognosis of Ewing's sarcoma originating bones and joints is much worse than that of Ewing's sarcoma of other parts of human body. Early screening and diagnosis seem to be more important among people at high risk of having Ewing's sarcoma of bones and joints.

The development of diagnostic methods based on molecular techniques has had a great effect, because typical chromosomal translocations are commonly detected in Ewing's sarcoma tissue. The reverse transcription-polymerase chain reaction (RT-PCR)

Table 1. Characteristics of patients with Ewing's sarcoma of the bones and joints

\begin{tabular}{|c|c|c|}
\hline \multirow{2}{*}{ Characteristic } & \multicolumn{2}{|c|}{ Total } \\
\hline & $\mathbf{N}$ & $\%$ \\
\hline Patients & 397 & 100 \\
\hline \multicolumn{3}{|l|}{ Sex } \\
\hline Female & 148 & 37.2 \\
\hline Male & 249 & 62.8 \\
\hline \multicolumn{3}{|l|}{ Age, years } \\
\hline$<18$ & 251 & 63.2 \\
\hline$\geq 18$ & 146 & 36.8 \\
\hline Mean (with standard deviation) & $18.5(12.4)$ & - \\
\hline Median & 17.8 & - \\
\hline \multicolumn{3}{|l|}{ Race } \\
\hline White & 351 & 88.4 \\
\hline Black & 15 & 3.8 \\
\hline Other & 31 & 7.8 \\
\hline \multicolumn{3}{|l|}{ Tumor site } \\
\hline Extremity & 219 & 55.1 \\
\hline Spine and pelvis & 178 & 44.9 \\
\hline \multicolumn{3}{|l|}{ Tumor grade } \\
\hline Low & 19 & 4.8 \\
\hline High & 378 & 95.2 \\
\hline \multicolumn{3}{|l|}{ Tumor size } \\
\hline$\leq 10$ & 173 & 43.6 \\
\hline$>10$ & 224 & 56.4 \\
\hline Mean (with standard deviation) & $10.3(5.3)$ & - \\
\hline Median & 9.6 & - \\
\hline \multicolumn{3}{|l|}{ Treatment } \\
\hline Surgery with radiotherapy & 86 & 21.6 \\
\hline Surgery alone & 139 & 35.1 \\
\hline Radiotherapy alone & 102 & 25.7 \\
\hline None & 70 & 17.6 \\
\hline
\end{tabular}

and fluorescent in-situ hybridization (FISH) are the most frequent measures applied in fusion gene analysis.

It has been reported that the $\mathrm{t}(11 ; 22)$ ( $\mathrm{q} 24 ; \mathrm{q} 12)$ translocation is found in $85 \%$ of cases of Ewing's sarcoma. ${ }^{11,12}$ Yang et al. ${ }^{13}$ further ascertained that FISH and RT-PCR could be applied as reliable molecular diagnostic approaches in cases of Ewing's sarcoma,

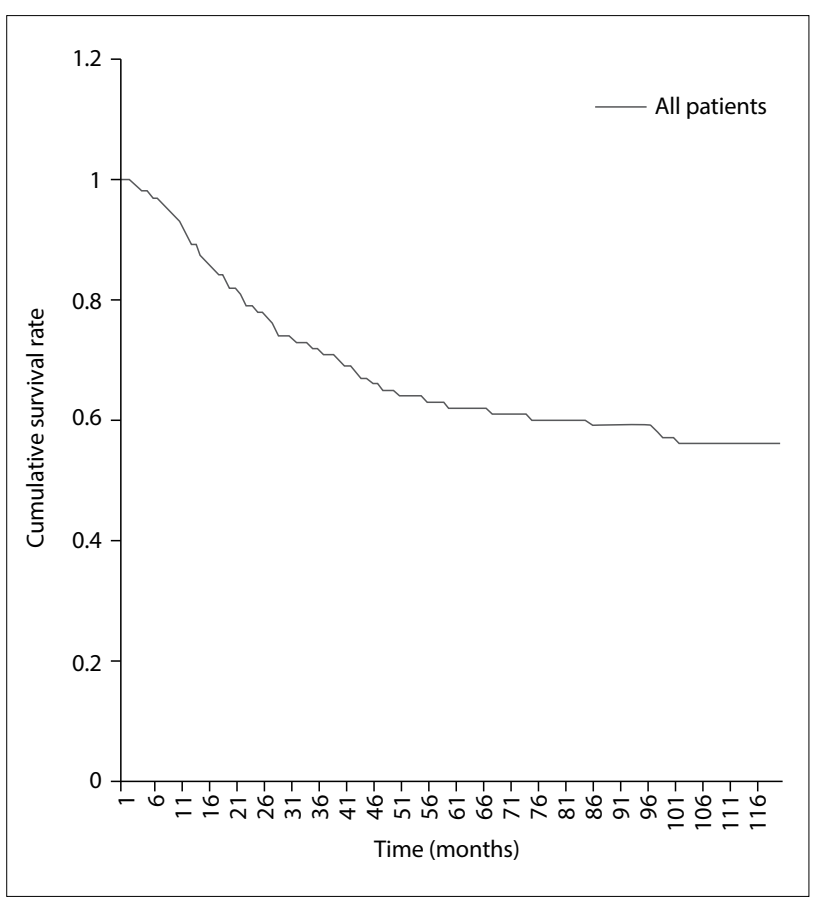

Figure 2. Kaplan-Meier survival curve for all patients.

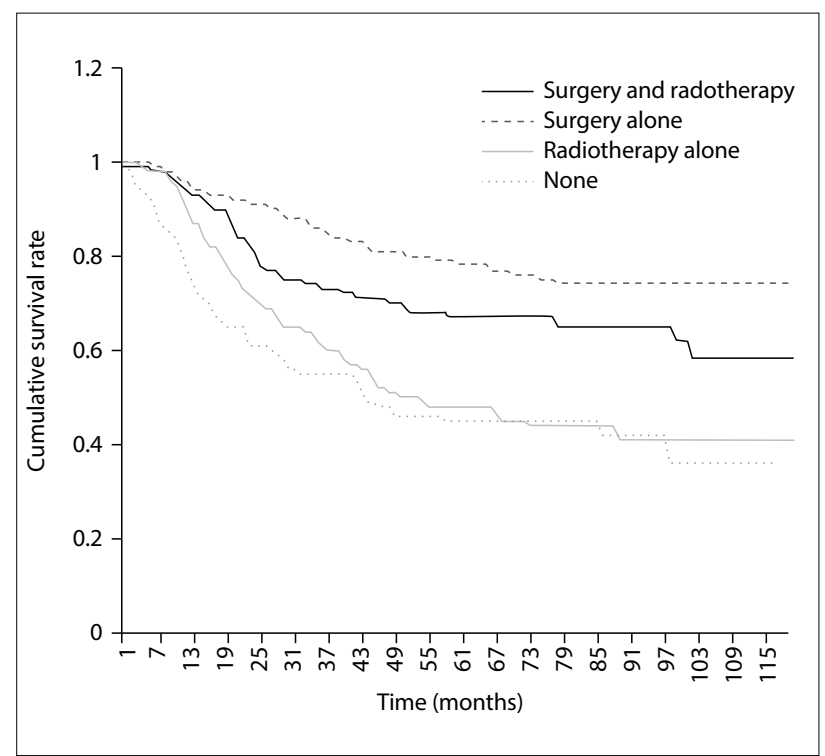

Figure 3. Kaplan-Meier survival curves for all patients, stratified according to type of treatment. 
and that FISH displayed features of greater sensitivity and stability. Furthermore, in a meta-analysis on 1,412 cases, Li et al. ${ }^{14}$ declared that high levels of serum lactate dehydrogenase (LDH) presaged lower DSS among patients with Ewing's sarcoma. However, information on these specific molecular indicators is not documented in the SEER database.

The influence of age on survival has always been a matter of debate, with contrary outcomes reported from different studies.

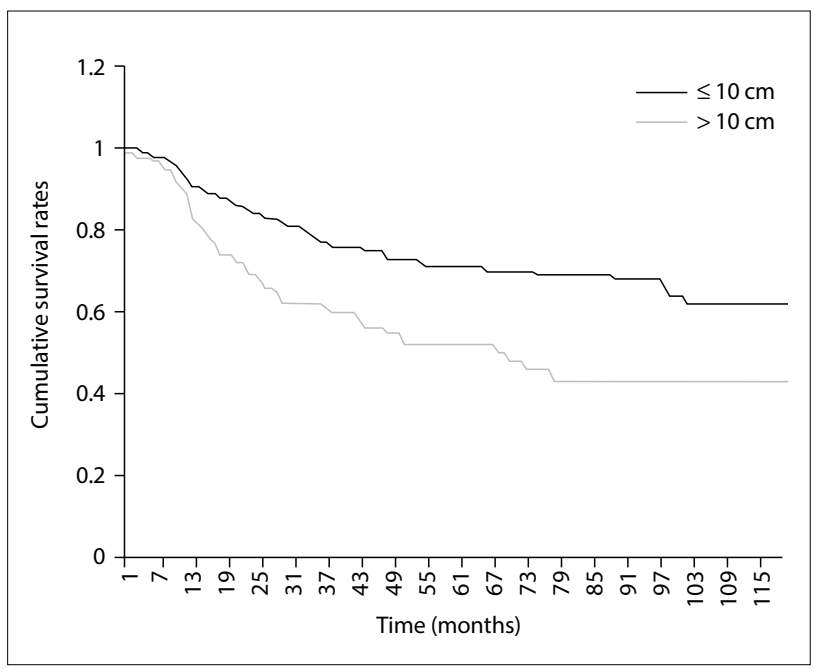

Figure 4. Kaplan-Meier survival curves for all patients, stratified according to tumor size.

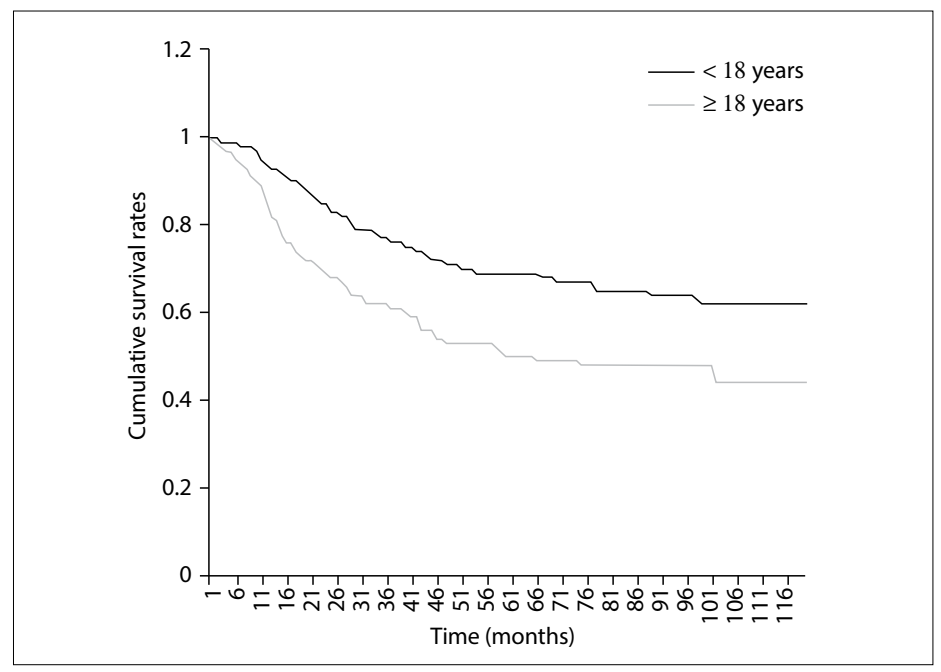

Figure 5. Kaplan-Meier survival curves for all patients, stratified according to age.

Table 2. Cox model with hazard ratios and $95 \%$ confidence intervals for mortality associated with covariates, among patients with Ewing's sarcoma of bones and joints

\begin{tabular}{|c|c|c|c|c|c|c|}
\hline \multirow{2}{*}{ Variable } & \multicolumn{3}{|c|}{ Crude } & \multicolumn{3}{|c|}{ Adjusted* } \\
\hline & HR & $(95 \% \mathrm{CI})$ & P-value & HR & $(95 \% \mathrm{Cl})$ & P-value \\
\hline \multicolumn{7}{|l|}{ Sex } \\
\hline Female & 1 & (reference) & & 1 & (reference) & \\
\hline Male & 1,47 & $(1.12-1.93)$ & $<0.05$ & 1.19 & $(0.98-1.71)$ & 0.074 \\
\hline \multicolumn{7}{|l|}{ Age } \\
\hline$<18$ years & 1 & (reference) & & 1 & (reference) & \\
\hline White & 1 & (reference) & & 1 & (reference) & \\
\hline Black & 1.68 & $(1.27-2.07)$ & $<0.05$ & 1.52 & $(1.37-1.86)$ & 0.075 \\
\hline Other & 0.63 & $(0.49-1.53)$ & 0.38 & 0.91 & $(0.68-1.74)$ & 0.84 \\
\hline \multicolumn{7}{|l|}{ Tumor site } \\
\hline Extremity & 1 & (reference) & & 1 & (reference) & \\
\hline High & 2.37 & $(0.77-4.29)$ & 0.81 & - & - & - \\
\hline \multicolumn{7}{|l|}{ Tumor size } \\
\hline$\leq 10 \mathrm{~cm}$ & 1 & (reference) & & 1 & (reference) & \\
\hline$>10 \mathrm{~cm}$ & 2.01 & $(1.46-2.77)$ & $<0.05$ & 1.78 & $(1.34-2.56)$ & $<0.05$ \\
\hline \multicolumn{7}{|l|}{ Treatment } \\
\hline Radiotherapy with surgery & 1 & (reference) & & 1 & (reference) & \\
\hline Surgery alone & 0.61 & $(0.42-0.92)$ & $<0.05$ & 0.51 & $(0.38-0.89)$ & $<0.05$ \\
\hline Radiotherapy alone & 1.66 & $(1.24-2.51)$ & $<0.05$ & 1.61 & $(1.10-2.39)$ & $<0.05$ \\
\hline None & 2.27 & $(1.53-3.38)$ & $<0.05$ & 1.86 & $(1.23-2.58)$ & $<0.05$ \\
\hline
\end{tabular}

$\mathrm{HR}=$ hazard ratio; $\mathrm{Cl}=$ confidence interval. *Adjusted for sex, age, race, tumor site, tumor size and treatment. 
In our study, most of the patients included were young, i.e. under 18 years of age, and the median age of our cohort was 17.8 years at the time of diagnosis. This was almost identical to the results reported previously in the worldwide literature, i.e. that most cases of Ewing's sarcoma not surprisingly emerged before the second decade of life, and that younger patients were likely to have a better prognosis. ${ }^{15-17}$ Regarding the reasons for this phenomenon, Lee et al. ${ }^{18}$ and Grevener et al. ${ }^{16}$ found that fewer cases among adult patients were treated with chemotherapy. Moreover, elderly patients were more likely to have several comorbidities, including diabetes mellitus hypertension or secondary cancers, which made the situation much more complex.

Tumor size was considered to be a prognostic indicator in our study. We found that the mean size was $10.3 \mathrm{~cm}$, which was almost consistent with the results declared in previous studies. We noticed that sizes larger than $10 \mathrm{~cm}$ were associated with a negative impact on DSS. However, there is no consensus regarding any critical cutoff size that might indicate a completely different prognosis for this disease. ${ }^{19-23}$ In a study on 182 patients, Fizazi et al. ${ }^{24}$ found that tumor size greater than $10 \mathrm{~cm}$ was an independent prognostic factor for survival. Canter ${ }^{25}$ also recommended that patients with tumors larger than $10 \mathrm{~cm}$ should accept neoadjuvant chemotherapy and investigational therapies, because they were at a high risk of relapse and disease-specific death.

Even so, several studies have asserted that $8 \mathrm{~cm}$ might be a more appropriate boundary value. In a retrospective analysis on 220 patients at St. Jude Children's Research Hospital, RodriguezGalindo et al. ${ }^{23}$ found that neoplasm size larger than $8 \mathrm{~cm}$ affected survival adversely.

In our analysis, tumors arising from the spine and pelvis were an independent factor for poorer survival. The proportion of the patients who accepted surgery was $56.0 \%$, while $47.5 \%$ received radiotherapy. We found that surgery alone, radiotherapy alone, and no treatment were independent risk factors.

There are several explanations for this phenomenon. Oberlin et al. ${ }^{26}$ asserted that it was recommendable that smaller and more peripheral tumors should be dealt with through surgical resection, while larger and more central unresectable entities should be managed with radiotherapy. On the other hand, Granowetter et al. ${ }^{27}$ pointed out that radiotherapy was not appropriate for patients in whom there was no proof of microscopic remainders of malignant tissue after they underwent operations.

Normally, it is accepted that surgery will provide a decisive partial cure. Only when the neoplasm is unresectable or after palliative surgery should radiation therapy be considered. Such patients' prognoses have been found to be relatively much worse than those of patients who underwent surgery alone..$^{10,28}$

In a retrospective study on 512 cases, Bacci et al..$^{29}$ concluded that surgical resection is more ideal than radiation therapy for patients with Ewing's sarcoma of the extremities for whom adequate surgical margins can be achieved. In cases of insufficient surgical margins, high-dose radiotherapy is recommended, while reduceddose radiotherapy is ineffective.

Furthermore, the main population affected by Ewing's sarcoma of the bones and joints consists of young people, mostly in their teenage years. For these individuals, excessive exposure to radiation may result in retardation of the development of bones and other organs. This may have side effects of greater severity than those of surgery, which may produce less morbidity. Although surgery is the practice most often used for local control, there are very few randomized controlled trials directly comparing the effects of surgery with those of radiotherapy, and the relative positions of these techniques remain contentious. ${ }^{28}$

In addition, race, sex and tumor grade were not independent factors after adjusting for different variables in our Cox multivariate regression model. On the other hand, these valuables were reported to be independent risk factors in relation to other bone cancers in some previous studies. ${ }^{19,20,22,30,31}$ Further study regarding whether these factors should be considered as independent risk indicators for the prognosis of patients with Ewing's sarcoma of the bones and joints is needed.

Our analysis was based on the data documented in the SEER database, which means that we need to acknowledge that there were some limitations relating to our study. Firstly, some variables including data on comorbidities, surgical margins, extent of surgical resection, tumor recrudescence and use of targeted therapy in managing this cancer were missing or not recorded in the database. Secondly, because of the principle of anonymity in the SEER Program, it was impossible for us to contact the patients in order to gain additional information. Thirdly, it also should not be ignored that because of the existence of confounders, the consequences deduced from a retrospective analysis would normally be of lower methodological grade than those from randomized controlled trials. Finally, we were unable to evaluate some specific molecular indicators, such as Ewing's Sarcoma-Friend leukemia integration 1 transcription factor (EWS-FLI1) and serum LDH, which help in making an early diagnosis and in judging the prognosis.

In spite of these limitations, use of the SEER Program database has significant advantages, in that it provides possibilities for conducting studies of this nature based on large populations suffering from rare types of cancer.

\section{CONCLUSION}

In conclusion, the contemporary five-year DSS rate of Ewing's sarcoma of the bones and joints was $62.47 \%$. Age $\geq 18$ years, tumors originating in the spine and pelvis, tumor size $>10 \mathrm{~cm}$, receiving radiotherapy alone and no treatment were independent risk factors for poor DSS, while surgery alone was an 
independent protective factor for better survival. Further investigations combining multiple fields of the gene modulation and molecular mechanism- are expected to elucidate better treatment strategies for cases of Ewing's sarcoma of the bones and joints.

\section{REFERENCES}

1. Uyeturk U, Helvaci K, Demirci A, et al. Clinical outcomes and prognostic factors of adult's Ewing sarcoma family of tumors: single center experience. Contemp Oncol (Pozn). 2016;20(2):141-6.

2. Biswas B, Bakhshi S. Management of Ewing sarcoma family of tumors: Current scenario and unmet need. World J Orthop. 2016;7(9):527-38.

3. Delattre O, Zucman J, Melot T, et al. The Ewing family of tumors--a subgroup of small-round-cell tumors defined by specific chimeric transcripts. N Engl J Med. 1994;331(5):294-9.

4. Esiashvili N, Goodman M, Marcus RB Jr. Changes in incidence and survival of Ewing sarcoma patients over the past 3 decades: Surveillance Epidemiology and End Results data. J Pediatr Hematol Oncol. 2008;30(6):425-30.

5. Riggi N, Stamenkovic I. The Biology of Ewing sarcoma. Cancer Lett. 2007;254(1):1-10.

6. Rodriguez-Galindo C, Spunt SL, Pappo AS. Treatment of Ewing sarcoma family of tumors: current status and outlook for the future. Med Pediatr Oncol. 2003;40(5):276-87.

7. Teicher BA, Bagley RG, Rouleau C, et al. Characteristics of human Ewing/ PNET sarcoma models. Ann Saudi Med. 2011;31(2):174-82.

8. ESMo Guidelines Working Group, Saeter G. Ewing's sarcoma of bone: ESMO clinical recommendations for diagnosis, treatment and followup. Ann Oncol. 2007;18 Suppl 2:ii79-80.

9. National Cancer Institute. Surveillance, Epidemiology, and End Results Program. SEER*Stat Installation. Available from: https://seer.cancer.gov/ seerstat/software/. Accessed in 2017 (Oct 6).

10. National Cancer Institute. Ewing Sarcoma Treatment (PDQ ${ }^{\oplus)}$-Health Professional Version; 2002. Available from: https://www.cancer.gov/ types/bone/hp/ewing-treatment-pdq. Accessed in 2017 (Sep 29).

11. Delattre O, Zucman J, Plougastel B, et al. Gene fusion with an ETS DNA-binding domain caused by chromosome translocation in human tumours. Nature. 1992;359(6391):162-5.

12. Turc-Carel C, Aurias A, Mugneret F, et al. Chromosomes in Ewing's sarcoma. I. An evaluation of 85 cases of remarkable consistency of t(11;22)(q24;q12). Cancer Genet Cytogenet. 1988;32(2):229-38.

13. Yang $Y$, Wang $H$, Wei $Y Y$, et al. [Application of fluorescence in-situ hybridization and reverse transcription-polymerase chain reaction in molecular diagnosis of Ewing's sarcoma and primitive neuroectodermal tumor]. Zhonghua Bing Li Xue Za Zhi. 2006;35(6):328-32.

14. Li S, Yang $Q$, Wang $H$, et al. Prognostic significance of serum lactate dehydrogenase levels in Ewing's sarcoma: A meta-analysis. Mol Clin Oncol. 2016;5(6):832-8.
15. Arshi A, Sharim J, Park DY, et al. Prognostic determinants and treatment outcomes analysis of osteosarcoma and Ewing sarcoma of the spine. Spine J. 2017;17(5):645-55.

16. Grevener K, Haveman LM, Ranft A, et al. Management and Outcome of Ewing Sarcoma of the Head and Neck. Pediatr Blood Cancer. 2016;63(4):604-10

17. Davenport JR, Vo KT, Goldsby R, West DC, DuBois SG. Conditional Survival and Predictors of Late Death in Patients with Ewing Sarcoma. Pediatr Blood Cancer. 2016;63(6):1091-5.

18. Lee J, Hoang BH, Ziogas A, Zell JA. Analysis of prognostic factors in Ewing sarcoma using a population-based cancer registry. Cancer. 2010;116(8):1964-73.

19. Ellis MA, Gerry DR, Neskey DM, Lentsch EJ. Ewing Sarcoma of the Head and Neck. Ann Otol Rhinol Laryngol. 2017;126(3):179-84.

20. Duchman KR, Gao Y, Miller BJ. Prognostic factors for survival in patients with Ewing's sarcoma using the surveillance, epidemiology, and end results (SEER) program database. Cancer Epidemiol. 2015;39(2):189-95

21. Ahrens S, Hoffmann C, Jabar S, et al. Evaluation of prognostic factors in a tumor volume-adapted treatment strategy for localized Ewing sarcoma of bone: the CESS 86 experience. Cooperative Ewing Sarcoma Study. Med Pediatr Oncol. 1999;32(3):186-95.

22. Karski EE, Mcllvaine E, Segal MR, et al. Identification of Discrete Prognostic Groups in Ewing Sarcoma. Pediatr Blood Cancer. 2016;63(1):47-53.

23. Rodriguez-Galindo C, Liu T, Krasin MJ, et al. Analysis of prognostic factors in ewing sarcoma family of tumors: review of St. Jude Children's Research Hospital studies. Cancer. 2007;110(2):375-84.

24. Fizazi K, Dohollou N, Blay JY, et al. Ewing's family of tumors in adults: multivariate analysis of survival and long-term results of multimodality therapy in 182 patients. J Clin Oncol. 1998;16(12):3736-43.

25. Canter RJ. Chemotherapy: Does Neoadjuvant or Adjuvant Therapy Improve Outcomes? Surg Oncol Clin N Am. 2016;25(4):861-72.

26. Oberlin O, Deley MC, Bui BN, et al. Prognostic factors in localized Ewing's tumours and peripheral neuroectodermal tumours: the third study of the French Society of Paediatric Oncology (EW88 study). Br J Cancer. 2001;85(11):1646-54.

27. Granowetter L, Womer R, Devidas M, et al. Dose-intensified compared with standard chemotherapy for nonmetastatic Ewing sarcoma family of tumors: a Children's Oncology Group Study. J Clin Oncol. 2009;27(15):2536-41.

28. DuBois SG, Krailo MD, Gebhardt MC, et al. Comparative evaluation of local control strategies in localized Ewing sarcoma of bone: a report from the Children's Oncology Group. Cancer. 2015;121(3):467-75.

29. Bacci G, Longhi A, Briccoli A, et al. The role of surgical margins in treatment of Ewing's sarcoma family tumors: experience of a single institution with 512 patients treated with adjuvant and neoadjuvant chemotherapy. Int J Radiat Oncol Biol Phys. 2006;65(3):766-72. 
30. Bacci G, Longhi A, Ferrari S, et al. Prognostic factors in non-metastatic Ewing's sarcoma tumor of bone: an analysis of 579 patients treated at a single institution with adjuvant or neoadjuvant chemotherapy between 1972 and 1998. Acta Oncol. 2006;45(4):469-75.

31. Jawad MU, Cheung MC, Min ES, et al. Ewing sarcoma demonstrates racial disparities in incidence-related and sex-related differences in outcome: an analysis of 1631 cases from the SEER database, 1973-2005. Cancer. 2009;115(15):3526-36.

Sources of funding: None

Conflict of interest: None

Date of first submission: July 30, 2017

Last received: September 11, 2017

Accepted: September 23, 2017

\section{Address for correspondence:}

Liao-Bin Chen

Department of Orthopedic Surgery, Zhongnan Hospital, Wuhan

University

169 Donghu Road

Wuhan - China

Tel. + 86-027-67872960

E-mail: liaobinchen@163.com 